\title{
Nintedanib y pirfenidona en el tratamiento farmacológico de la fibrosis pulmonar idiopática: perspectivas actuales y futuras.
}

\author{
Javier Leonardo Galindo ${ }^{1}$, Carlos Andrés Celis ${ }^{1,2}$ y Mayra Mejía \\ ${ }^{1}$ Unidad de Neumología, Hospital Universitario San Ignacio. Bogotá, Colombia. \\ ${ }^{2}$ Pontificia Universidad Javeriana. Bogotá, Colombia. \\ ${ }^{3}$ Unidad de Enfermedades Pulmonares Intersticiales. Instituto Nacional de \\ Enfermedades Respiratorias, Ismael Cosío Villegas. Ciudad de México, México.
}

Palabras clave: fibrosis pulmonar idiopática; enfermedades pulmonares intersticiales; terapéutica; tratamiento farmacológico.

Resumen. La fibrosis pulmonar idiopática es una enfermedad progresiva y fatal. En los últimos años se han identificado algunos factores involucrados en el desarrollo de la lesión del epitelio alveolar y en el remodelado anormal que conducen a la fibrosis, los cuales han sido evaluados para el desarrollo de tratamientos específicos. Dos terapias modificadoras de la enfermedad, pirfenidona y nintedanib, han demostrado reducir la progresión de la enfermedad, en términos de disminución de la capacidad vital forzada, con un efecto limítrofe sobre la mortalidad. Estos medicamentos han sido aprobados para su uso en pacientes con deterioro leve a moderado de la función pulmonar. Estudios de la vida real han evaluado estos tratamientos en poblaciones no representadas en los ensayos clínicos, con beneficios, seguridad y tolerancia similares. Los medicamentos difieren en su perfil de seguridad, pero ninguno ha demostrado ser mejor al otro en términos de eficacia. La decisión de comenzar estos tratamientos debe ponderar la gravedad de la enfermedad y las expectativas y preferencias del paciente. El tratamiento farmacológico combinado posiblemente será el tratamiento estándar en el futuro, pero nuevos estudios deberán evaluar su eficacia. 


\title{
Nintedanib and pirfenidone for the treatment of idiopathic pulmonary fibrosis: current and future perspectives.
}

\author{
Invest Clin 2018; 59 (4): 369 - 385
}

Key words: idiopathic pulmonary fibrosis; interstitial lung diseases; therapeuties; drug therapy.

\begin{abstract}
Idiopathic pulmonary fibrosis is a progressive and fatal disease. In recent years, some factors involved in the pathophysiology of the alveolar epithelial injury and in the abnormal remodeling that lead to fibrosis have been identified, some factors involved in the pathophysiology of the alveolar epithelial injury and in the abnormal remodeling that lead to fibrosis have been identified, some of them have been evaluated for the development of specific treatments. Two disease-modifying therapies, pirfenidone and nintedanib, have demonstrated to reduce the progression of the disease, in terms of decline in forced vital capacity, with a borderline effect on mortality. These drugs have been approved for patients with mild to moderate impairment in pulmonary function tests. Real-life studies have evaluated these treatments in populations not represented in clinical trials, with similar benefits, safety and tolerance. Pirfenidone and nintedanib have different safety profiles, but none is better in terms efficacy. Decision to start treatment should weight the severity of the disease and the patient's expectations and preferences. Combined drug treatment possibly will be the standard of treatment in the future, but further studies must assess its efficacy.
\end{abstract}

Recibido 30-04-2018 Aceptado 25-10-2018

\section{INTRODUCCIÓN}

La fibrosis pulmonar idiopática (FPI) es la neumonía intersticial idiopática más frecuente en el mundo. Se caracteriza por tener un curso heterogéneo, irreversible, progresivo e impredecible, asociado con una morbilidad significativa y un mal pronóstico después del diaǵnóstico (1). Existe una creciente evidencia acerca de que la enfermedad se origina por la interacción entre una expresión variable de polimorfismos genéticos, cambios relacionados con el envejecimiento celular, y exposiciones a ciertos factores ambientales, como el tabaquismo, los polvos industriales, la microaspiración gástrica crónica, infecciones virales y, posiblemente, alteraciones en el microbioma pul- monar $(2,3)$. Las lesiones producidas por las exposiciones repetitivas, activan de manera aberrante a las células epiteliales alveolares de individuos genéticamente susceptibles, promoviendo la apoptosis del epitelio, el reclutamiento de células mesenquimales y el aumento de la permeabilidad vascular (Fiǵ. 1). La interacción epitelial/mesenquimal no regulada conduce a la secreción de una variedad de citocinas profibróticas, metaloproteinasas y mediadores procoagulantes, que promueven la migración y proliferación no controlada de fibroblastos, la diferenciación a miofibroblastos y la fibrosis en la matriz extracelular $(2,4)$.

Dado que en la FPI la inflamación es escasa o inexistente, el uso de inmunosupresores no ha resultado ser útil y, por el contra- 


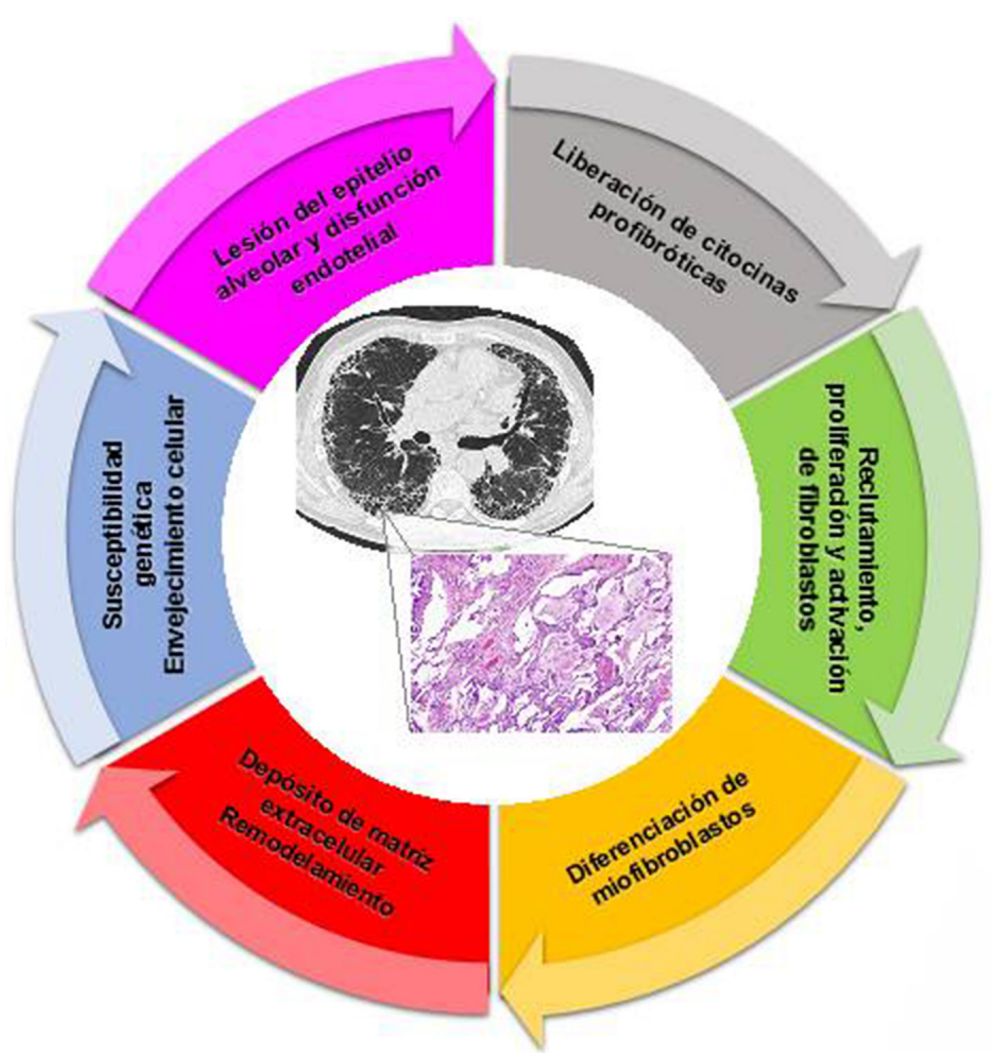

Fig.1. Mecanismos propuestos en la patogénesis de la fibrosis pulmonar idiopática. El humo del cigarrillo, los polvos industriales, el reflujo gastroesofáǵico o las infecciones virales pueden provocar la activación de las células epiteliales alveolares de individuos con susceptibilidad particular para desarrollar la fibrosis (por ejemplo, edad, genética), lo que produce una reparación aberrante y una acumulación excesiva de matriz extracelular.

rio, resulta ser lesivo. El estudio PANTHER demostró que la triple terapia, consistente en la combinación de prednisona, azatioprina y N-acetilcisteína (NAC), aumentaba la mortalidad y las admisiones hospitalarias (5). Recientemente, dos medicamentos modificadores de la enfermedad, pirfenidona y nintedanib, han sido aprobados para el tratamiento de FPI. Estos fármacos denominados "antifibróticos" disminuyen la tasa de progresión de la enfermedad en pacientes con alteración leve a moderada en las pruebas de función pulmonar (Tabla I). Esta revisión hace un recuento de la evidencia publicada sobre estos medicamentos y señala pautas para su empleo.

\section{Pirfenidona}

La pirfenidona (5-metil-1-fenil-2-[1H]piridona), es un medicamento con mecanismos de acción no completamente conocidos (6). Se ha propuesto que la pirfenidona inhibe la expresión de citocinas profibróticas, como el factor de crecimiento transformante- $\beta$, el factor de crecimiento derivado de las plaquetas (PDGF) y el factor de necrosis tumoral- $\alpha$, y que además tiene una acción antioxidante por disminución de la actividad de la mieloperoxidasa y la superóxido dismutasa $(6,7)$. Las concentraciones séricas máximas luego de la administración oral se alcanzan en 30 a 60 minutos en ayuno y en 3,5 horas con la ingesta de alimentos; su vida media es 


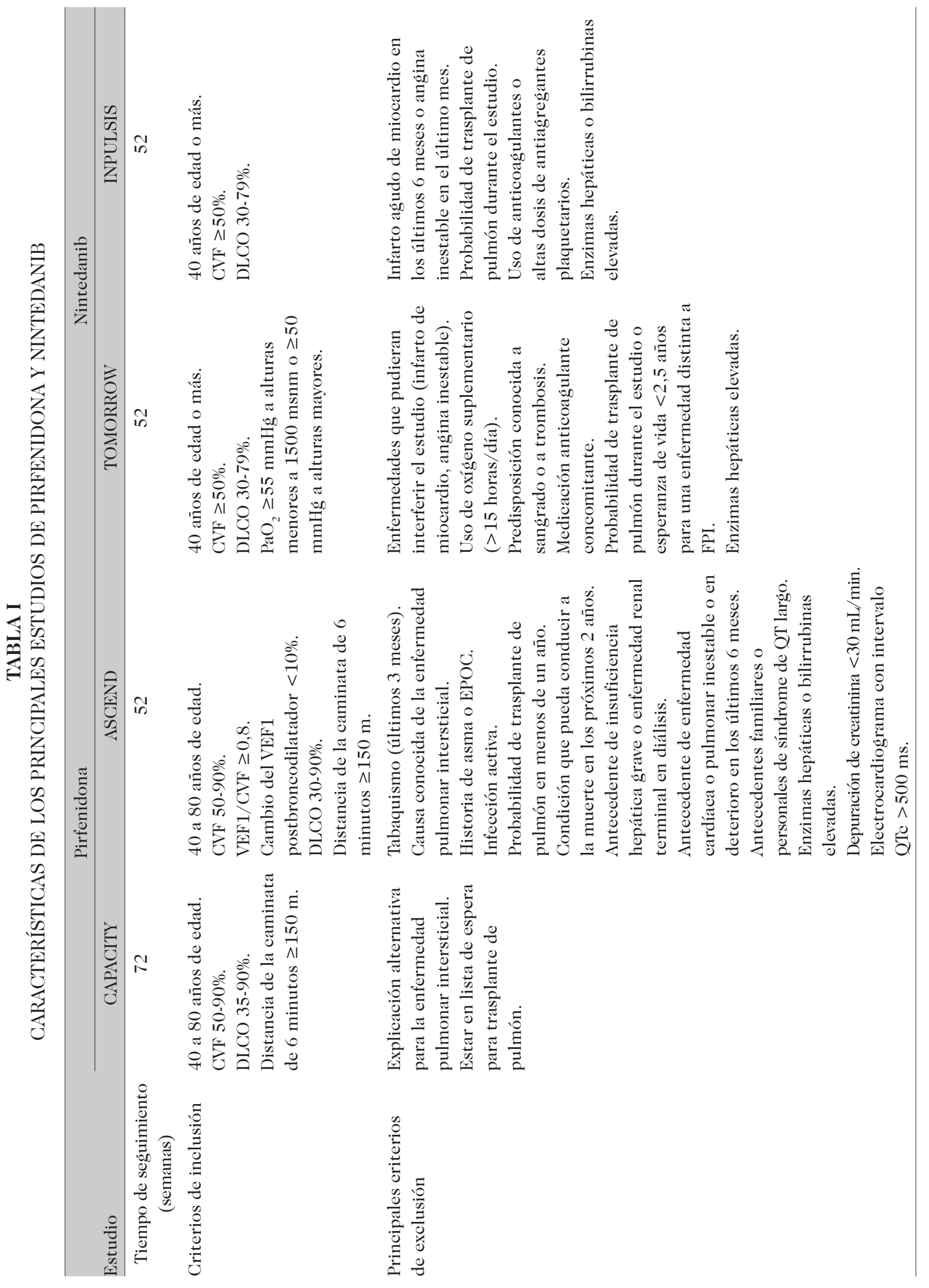




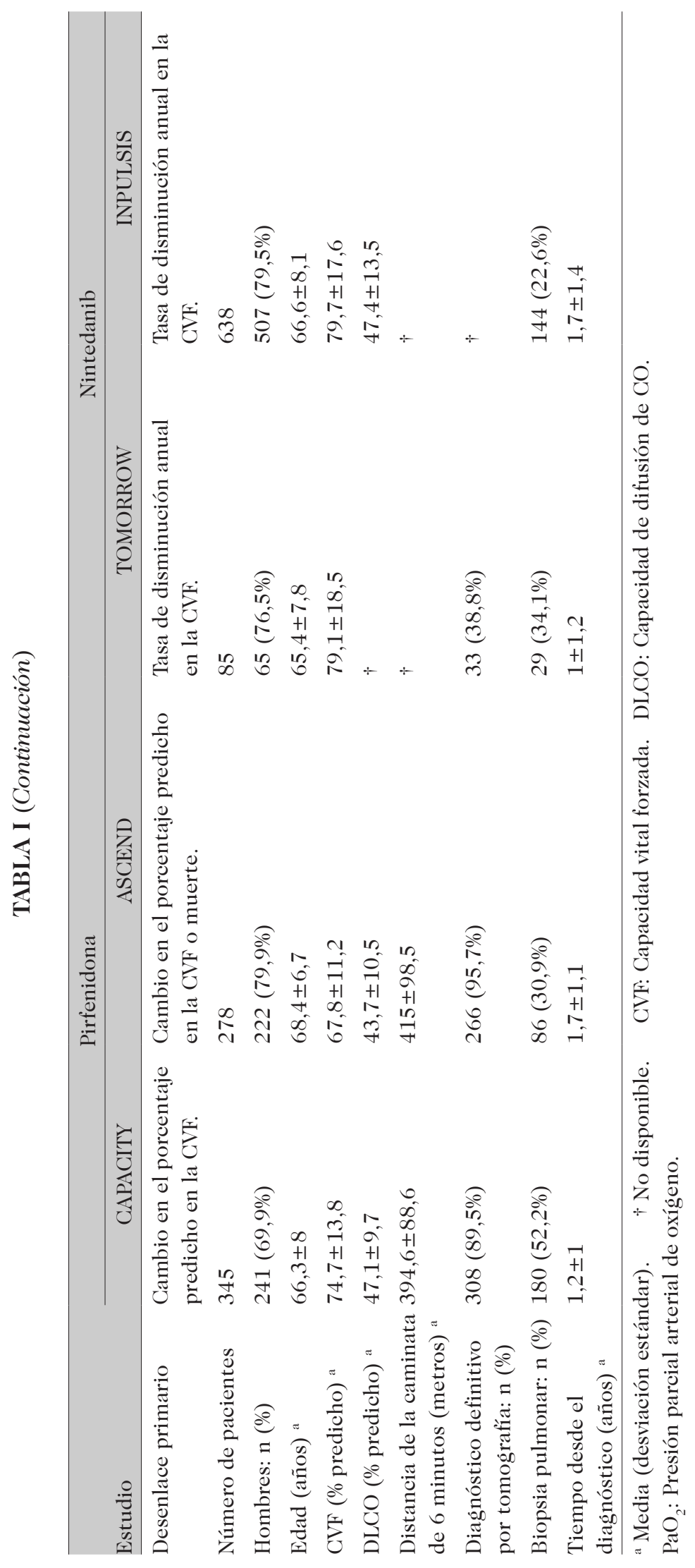


de 2,4 horas con el consumo de alimentos y 2,9 horas en ayuno (6). La pirfenidona es metabolizada principalmente por el citocromo CYP1A2 (70 a 80\%), con contribuciones menores de otros citocromos, como CYP2C9, CYP2C19, CYP2D6 y CYP2E1; los medicamentos metabolizados por estos citocromos, como amiodarona, ciprofloxacina, fluvoxamina o propafenona, deben utilizarse con precaución ya que pueden aumentar los niveles séricos de pirfenidona (6). La dosis del medicamento se titula a partir de una cápsula de $267 \mathrm{mg}$ tres veces al día durante una semana, luego dos cápsulas tres veces al día durante la siguiente semana y finalmente tres cápsulas tres veces al día $(6,7)$.

Cinco estudios clínicos controlados con placebo han evaluado la eficacia y seguridad de pirfenidona (8-11). El primer estudio fase 2, realizado en Japón, tuvo como objetivo evaluar el cambio en la saturación más baja de oxígeno registrada durante una prueba de ejercicio de 6 minutos en estado estacionario; la pirfenidona a dosis de 1800 mǵ diarios logró mejores saturaciones a los 9 meses respecto al placebo $(p=0,0305)$ (8). Desenlaces secundarios, como la muerte por exacerbaciones y la disminución de la capacidad vital forzada (CVF), también fueron menores con el uso de pirfenidona. Un segundo estudio de Japón fase 3, comparó las dosis de 1200 y de $1800 \mathrm{mg}$ diarios de pirfenidona contra un grupo placebo, demostrando una disminución en el declinamiento en la CVF después de 52 semanas en los pacientes con la dosis más alta de pirfenidona en comparación con el placebo (-0,09 vs. -0,16 L/año; $p=0,04)$; no se revalidó el efecto sobre las exacerbaciones observado en el primer estudio (9).

El estudio CAPACITY se compuso de dos ensayos concurrentes fase 3. El estudio 004 fue diseñado para evaluar dos dosis de pirfenidona (2403 mg/día y 1197 mg/día) contra a placebo, mientras que el estudio 006 evaluó la dosis de $2403 \mathrm{mg}$ diarios contra a placebo (Tabla II) (10). En el estudio 004, la dosis de $2403 \mathrm{mg}$, redujo la tasa de decli- namiento en la CVF en comparación al placebo, pero el estudio 006 falló en demostrar una diferencia en el cambio en la CVF entre los grupos evaluados. En el análisis combinado de ambos estudios se demostró un efecto benéfico de la pirfenidona en la reducción en la CVF $(-8,5 \%$ vs. $-11 \% ; p=0,005)$, la supervivencia libre de progresión y la distancia de la caminata de 6 minutos. Los eventos adversos más reportados fueron gastrointestinales (náuseas 36\% y dispepsia 19\%) y cutáneos (erupción 32\% y fotosensibilidad 12\%); estos eventos condujeron a la interrupción del tratamiento en el 15\% de los pacientes. Con estos resultados el medicamento fue aprobado para su uso en la Unión Europea, pero debido a los resultados discordantes en el desenlace primario la Administración de Alimentos y Medicamentos (FDA), en los Estados Unidos, solicitó un estudio adicional.

El estudio ASCEND realizó una distribución aleatoria de pacientes para recibir pirfenidona a dosis de 2403 mǵ por día o placebo (11). El promedio de disminución en la GVF fue menor en quienes recibieron pirfenidona, así como la proporción de pacientes que tuvieron una disminución igual o mayor de $10 \%$ en la CVF o muerte. No hubo diferencias en la mortalidad por todas las causas o la mortalidad relacionada con la FPI. Los eventos adversos más frecuentes fueron gastrointestinales y cutáneos; los eventos adversos llevaron a la interrupción del tratamiento en el $14,4 \%$ de los pacientes con pirfenidona.

En el análisis combinado de los estudios ASCEND y CAPACITY, la pirfenidona después de 52 semanas de tratamiento, redujo el riesgo de una disminución mayor o igual a $10 \%$ en la CVF o de muerte (HR 0,48; IC 95\% 0,37-0,63), la mortalidad por todas las causas (HR 0,52; IC 95\% 0,31-0,87), la mortalidad relacionada con la FPI (HR 0,35; IC 95\% 0,17-0,72), la mortalidad emergente en el tratamiento relacionada con la FPI (HR 0,32; IC 95\% 0,14-0,76) y las hospitalizaciones por causas respiratorias (HR 0,52; IC 95\% 0,36-0,77) (11-14). A 120 semanas, 


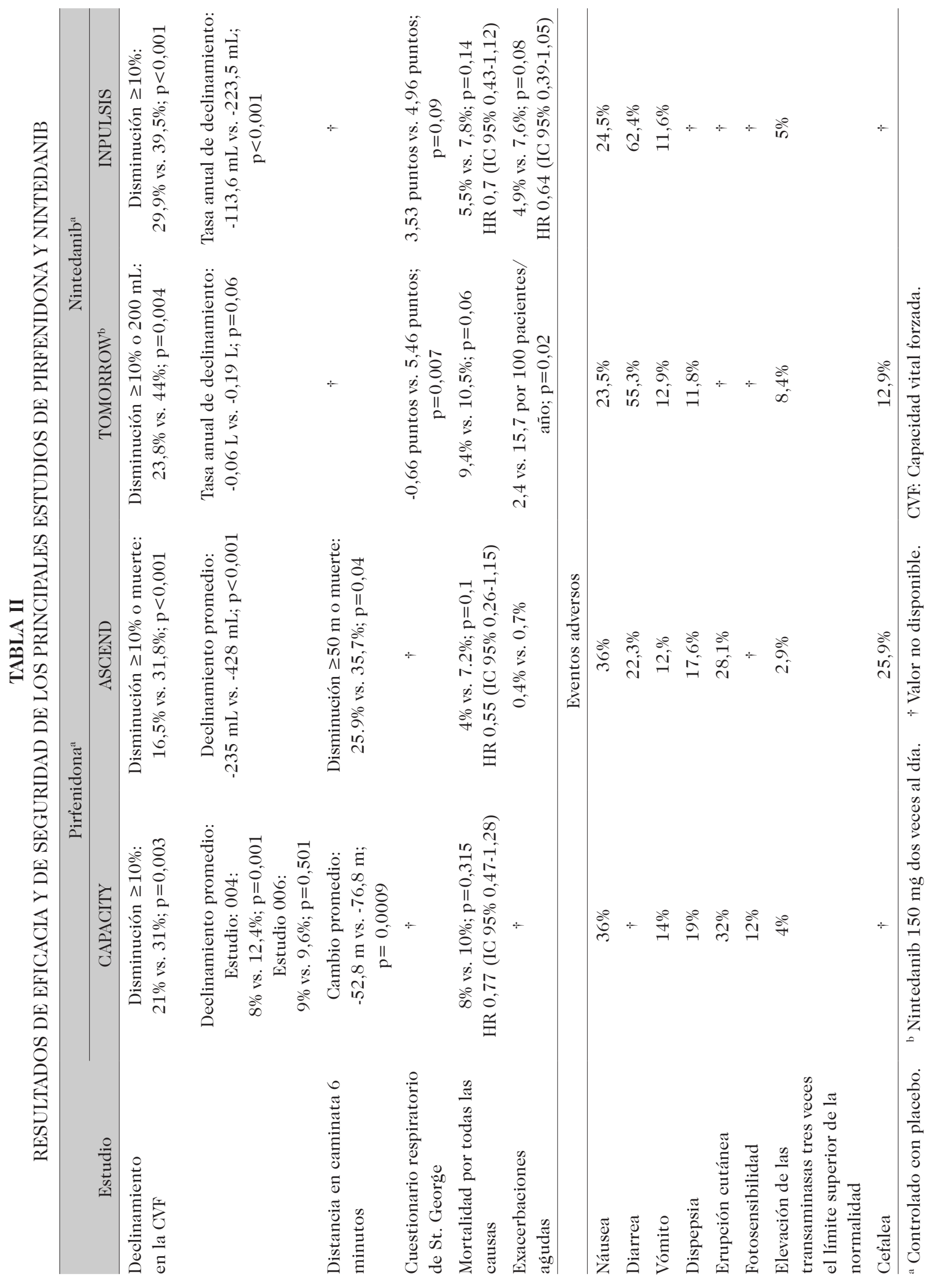


se mantuvieron diferencias con respecto a la mortalidad por todas las causas, la mortalidad relacionada con la FPI y la mortalidad emergente en el tratamiento relacionada con la FPI (13). Un metanálisis de los cineo ensayos clínicos publicados, mostró un efecto benéfico consistente de la pirfenidona en la mortalidad (15). Modelos predictivos basados en dichos datos han estimado que la pirfenidona podría aumentar la esperanza de vida promedio en 2,47 años (IC 95\% 1,264,17) (16).

La pirfenidona tiene beneficios similares en pacientes con función pulmonar basal preservada (CVF igual o mayor de $80 \%$ ), apoyando el inicio del tratamiento en la FPI temprana (17). Por otra parte, el uso continuo de pirfenidona en pacientes que experimentan un descenso igual o mayor de $10 \%$ en la CVF al sexto mes de tratamiento, resulta en un menor riesgo de deterioro posterior en la CVF o muerte (5,9\% vs. $27,9 \%$; $p=0,009$ ) (18). Estos hallazgoos sugieren un efecto potencial del tratamiento continuo con pirfenidona en pacientes con progresión de la enfermedad a pesar del tratamiento, por lo que la única razón para suspender el tratamiento sería el desarrollo de eventos adversos intolerables (19).

Los eventos adversos derivados del uso de pirfenidona se asocian con la concentración plasmática máxima del medicamento y la frecuencia se reduce luego del sexto mes de tratamiento, a excepción de la fotosensibilidad $(20,21)$. Los efectos más frecuentes son gastrointestinales (náuseas $37,6 \%$ y diarrea 28,1\%) y cutáneos (erupción cutánea 25\%) (21). Las molestias gastrointestinales podrían mejorar tomando la pirfenidona con las comidas, espaciando la ingesta de cada cápsula y utilizando aǵentes procinéticos o inhibidores de la bomba de protones $(20,22)$. Sin embargo, tanto el omeprazol como el lanzoprazol son inductores de CYP1A2, por lo que se aconseja usar esomeprazol o pantoprazol $(20,22)$. Para prevenir la fotosensibilidad los pacientes deben usar protector solar con factor +50 y evitar la exposición a la luz solar cerca a las horas de consumo del medicamento $(7,20)$. Pueden ser necesarias la reducción de la dosis o la interrupción temporal por una a dos semanas para el control de los eventos adversos $(7,20)$. La adición de NAC a pirfenidona aumenta la incidencia de fotosensibilidad y parece inducir a una disminución mayor en la CVF (23). Los pacientes con enfermedad más avanzada tienen un mayor riesgo de presentar eventos adversos (22).

\section{Nintedanib}

El nintedanib etanosulfonato fue originalmente diseñado como un medicamento antiangiogénico para el tratamiento del cáncer (4). El nintedanib bloquea el sitio de unión intracelular del ATP de múltiples receptores tirosina cinasa, como el receptor del factor de crecimiento vascular endotelial (VEGFR) 1, 2 y 3, el receptor del factor de crecimiento fibroblástico 1,2 y 3 y el receptor del PDGF $\alpha$ y $\beta(4,24)$. La dosis inicial de nintedanib es de $150 \mathrm{mg}$ dos veces al día. Logra concentraciones plasmáticas máximas en 2 a 4 horas luego de la ingesta, tiene una vida media de 9,5 horas y alcanza concentraciones estables en una semana $(7,24)$. Se metaboliza en el hígado por el citocromo CYP450 y, en menor medida, por CYP3A4 y P-glicoproteína $(7,24)$. Los inhibidores de CYP3A4 o P-ǵlicoproteína como eritromicina o ketoconazol pueden obligar a una reducción de la dosis de nintedanib, mientras que debería evitarse la coadministración de medicamentos inductores de CYP3A4 o Pglicoproteína como carbamazepina, feitoína o rifampicina (7). Se debe tener precaución en pacientes que reciben anticoagulantes o antiagregantes plaquetarios, debido al riesgo potencial de hemorragia que tiene el uso de inhibidores del VEGFR (24).

Tres estudios clínicos controlados con placebo han evaluado la eficacia y seguridad de nintedanib (Tabla II) $(25,26)$. El estudio fase 2 TOMORROW aleatorizó pacientes con FPI a cuatro diferentes dosis de nintedanib (50 $\mathrm{mg}$ una vez al día, $50 \mathrm{mg}$ dos veces al día, $100 \mathrm{mg}$ dos veces al día y $150 \mathrm{mg}$ dos 
veces al día) o placebo (25). La dosis de 150 mg dos veces al día redujo la tasa anual de cambio en la CVF en comparación con el placebo, con una menor incidencia de exacerbaciones agudas y una discreta mejoría en el cuestionario respiratorio de St. George. Los eventos adversos gastrointestinales fueron más comunes con la dosis de $150 \mathrm{mg}$ dos veces al día (diarrea $55,3 \%$ y náusea $23,5 \%$ ), así como los eventos adversos que llevaron a la suspensión del tratamiento.

Los estudios INPULSIS-1 e INPULSIS-2 fueron estudios fase 3 paralelos, que evaluaron el nintedanib $150 \mathrm{mg}$ dos veces al día contra placebo (26). La tasa anual ajustada de deterioro en la CVF fue cerca de la mitad con el uso de nintedanib respecto al placebo; solo en el INPULSIS-2 hubo un aumento en el tiempo a la primera exacerbación. No se encontraron diferencias en los desenlaces al analizar los datos por edad, sexo, raza, tabaquismo o evidencia de panal de abejas en la tomografía, aunque pareció existir un efecto más pronunciado en la prevención de exacerbaciones en los pacientes con CVF igual o menor de $70 \%$ del predicho basal (HR 0,52; IC 95\% 0,28-0,99) $(27,28)$. Los pacientes con función pulmonar preservada (CVF mayor de 90\%) obtuvieron una tasa similar de disminución de la función pulmonar, sugiriendo que los pacientes en estadios tempranos de la enfermedad podrían beneficiarse del tratamiento (29).

El análisis combinado de los resultados de los estudios TOMORROW e INPULSIS mostró que la tasa anual de cambio en la CVF fue de $-112,4 \mathrm{~mL} /$ año $(2,8 \%)$ con nintedanib y de -223,3 mL/año (6\%) con placebo $(p<0,0001)(30)$. El riesgo para la primera exacerbación fue menor con nintedanib (HR 0,53; IC 95\% 0,34-0,83), al igual que la mortalidad durante el tratamiento (HR 0,57; IC 95\% 0,34-0,97).

El estudio de extensión abierto de la cohorte TOMORROW reveló que la tasa de cambio anual en CVF con nintedanib 150 mg dos veces al día fue de $-125,4 \mathrm{~mL} /$ año y con nintedanib $50 \mathrm{mg}$ una vez al día fue de
-189,7 mL/año más allá de las 52 semanas de tratamiento, con una menor proporción de pacientes con exacerbaciones con la dosis más alta (12,9 vs. $25,9 \%)$ (31). INPULSISON, el estudio abierto de extensión de los estudios INPULSIS, incluyó pacientes con CVF igual o menor de 50\%, estos pacientes tuvieron un efecto similar en el cambio en la función pulmonar que los pacientes con CVF mayor de $50 \%$ (-62,3 vs. $-87,9 \mathrm{~mL}$, respectivamente) (32). Estos hallazgos sugieren un posible beneficio del tratamiento en pacientes con enfermedad grave.

En los estudios INPULSIS entre el 23,7 y el $25,2 \%$ de los pacientes tratados con nintedanib, abandonaron prematuramente el tratamiento, principalmente por la presencia de eventos adversos en 18,8 a $21 \%$ de los casos (26). La reducción de la dosis a $100 \mathrm{mg}$ dos veces al día fue necesaria por lo menos una vez durante el seguimiento en $27,9 \%$ de los pacientes, debido al desarrollo de eventos adversos; al finalizar del estudio $76,3 \%$ de los pacientes mantuvieron o retomaron la dosis de 150 dos veces al día $(26,33)$.

La hidratación y el uso de loperamida se recomienda para el manejo de la diarrea causada por nintedanib (33). La reducción de la dosis o la interrupción, temporal o definitiva, pueden ser necesarias, dependiendo de la gravedad o de la persistencia de los síntomas gastrointestinales (33).

\section{Estudios de efectividad de las terapias modificadoras de la enfermedad}

Los experimentos clínicos no siempre reflejan la práctica clínica diaria, los pacientes seleccionados tienden a tener menos comorbilidades y menor gravedad de la enfermedad evaluada. Por ejemplo, a pesar de la alta letalidad de la FPI (alrededor de $20 \%$ por año), la mortalidad reportada en los grupos placebo de los estudios de las terapias modificadoras de la enfermedad fue tan solo de 2,3 a $7,8 \%$ (34). Es necesario tener información de estudios de efectividad que incluyan el espectro total de la población afectada con FPI. 
El registro post-mercadeo más grande de pirfenidona, publicado en Japón, reportó la experiencia de 1371 pacientes con la dosis recomendada para la población japonesa de $1800 \mathrm{mg} /$ día (35). Al final del periodo de seguimiento, 61,6\% de los pacientes tuvo estabilidad en la función pulmonar y un $48,7 \%$ descontinuó el tratamiento por progresión de la enfermedad. La mayoría de los pacientes sufrió eventos adversos (64,6\%), principalmente gastrointestinales $(40,1 \%)$, y fotosensibilidad $(14,4 \%)$.

El estudio PASSPORT, que es un registro de seguridad de pirfenidona de Europa, presentó datos preliminares de dos años de seguimiento con pirfenidona en 530 pacientes (34). De 311 pacientes con eventos adversos, 85 interrumpieron el tratamiento y una tercera parte de los pacientes requirió ajustes de la dosis.

Un centro en el Reino Unido, publicó la experiencia de 351 pacientes tratados con pirfenidona y 124 pacientes con nintedanib (36). Los pacientes con pirfenidona tenía un promedio basal de CVF de 69\% (rango 37146\%) y DLCO (Capacidad de difusión de monóxido de carbono) de 42\% (rango 14$112 \%$ ), un $16 \%$ tenía enfisema. Setenta y ocho por ciento de los pacientes con pirfenidona experimentó eventos adversos, principalmente gastrointestinales (anorexia 17\% y náuseas $15 \%$ ), requiriendo la interrupción del tratamiento en $20 \%$ de los casos. Las razones para el inicio de nintedanib fueron tener una CVF mayor de $80 \%$ (55\% de los easos), una CVF menor de 50\% (10\% de los easos), o eventos adversos intolerables con el uso de pirfenidona ( $27 \%$ de los easos). Los pacientes con nintedanib, tenían un promedio basal CVF de $81 \%$ (rango 35-120\%) y DLCO de $44 \%$ (rango 13-78\%). Los eventos adversos más frecuentes con nintedanib fueron gastrointestinales (diarrea 24\% y náuseas 13\%), llevando a la suspensión del medicamento en $19 \%$ de los casos o a la reducción de la dosis en $15 \%$ de los casos.
En una cohorte de 63 pacientes tratados con pirfenidona en Alemania, $85 \%$ tuvo eventos adversos, llevando a la descontinuación del tratamiento en el 20\% de los casos (37). Dentro de esta cohorte, $17 \%$ de los pacientes tenía combinación con enfisema y un $30 \%$ evidencia ecocardiográfica de hipertensión pulmonar. La progresión de la enfermedad y los eventos adversos se vieron más frecuentemente en quienes recibían esteroides o NAC. El programa de uso compasivo de nintedanib en Alemania, seleccionó 62 pacientes no elegibles para el uso de pirfenidona o con deterioro a pesar de su uso (38). La información de este registro mostró que nintedanib estabilizó la CVF en 63\% de los pacientes luego de 6 meses de tratamiento. Los eventos adversos más frecuentes fueron diarrea (63\%) y pérdida de peso (50\%); fue requerida en el $34 \%$ de los pacientes la reducción de la dosis y en el 10\% la interrupción del tratamiento.

Un centro en Filadelfia, publicó su experiencia clínica con 129 pacientes tratados con pirfenidona y 57 con nintedanib (39). Entre los pacientes con pirfenidona los eventos adversos más comunes fueron náuseas $(26,4 \%)$ y erupción cutánea/fotosensibilidad $(14,7 \%)$, mientras que en los pacientes con nintedanib fueron diarrea $(52,6 \%)$ y náuseas $(29,8 \%)$. De los sujetos con pirfenidona $66,7 \%$ toleró el medicamento sin reducción de la dosis, en comparación a 52,6\% de los sujetos con nintedanib. La discontinuación del medicamento fue necesaria en $20,9 \%$ y $26,3 \%$ de los casos para pirfenidona y nintedanib, respectivamente. Respecto a las exacerbaciones agudas, hubo una tendencia no significativa a presentar menos casos $(10,5$ vs. $16,3 \%)$ y a tener un tiempo más prolongado antes de la primera exacerbación (media de 294 vs. 247 días) con nintedanib.

En general, en Latinoamérica es limitada la experiencia de uso de estos medicamentos por el costo y la disponibilidad de los fármacos, que varía en cada uno de los países 
de conformidad con la configuración de cada sistema de salud. Argentina publicó la información de 47 pacientes del programa de uso compasivo de nintedanib (40). La CVF basal promedio fue de $65,9 \pm 19,2 \%(21,3 \%$ de los pacientes tenía una CVF menor de 50\%) y la DLCO promedio fue de $38,7 \pm 3,1 \%$. Hubo escasos eventos adversos reportados, los más comunes fueron la pérdida de peso $(10,6 \%)$ y la diarrea $(8,5 \%)$.

La experiencia de los autores, en las observaciones individuales de los casos tratados, es que los eventos adversos se presentan en una frecuencia y severidad similar a la reportada en los estudios originales. La mayoría de los pacientes toleran los medicamentos, siempre que sean instruidos sobre sus posibles eventos adversos y estos se aborden acorde a las recomendaciones de los fabricantes. Parte de la experiencia de los autores con las terapias modificadoras de la enfermedad se obtuvo al participar en los protocolos de los estudios INPULSIS y ASCEND.

En resumen, los estudios de la vida real muestran que el nintedanib tiene una menor frecuencia de efectos adversos que la reportada en los estudios de eficacia. Aunque la pirfenidona es un medicamento bien tolerado, su descontinuación por intolerancia es más común en estudios de la vida real. Es importante destacar que dentro de estas experiencias, fueron incluidos pacientes con una mayor frecuencia de comorbilidades y gravedad de la FPI, que por sus características habían sido excluidos de los estudios clínicos. Las experiencias con terapias modificadoras de la enfermedad, muestran que la educación y el apoyo a los pacientes mejoran el manejo de los posibles eventos adversos (36).

\section{Recomendaciones para el tratamiento de la FPI}

Durante el abordaje de las enfermedades pulmonares intersticiales, siempre que se documente un patrón histológico o radiológico de neumonía intersticial usual, es imprescindible una búsqueda exhaustiva de causas secundarias. Una vez que el patrón de neumonía intersticial usual se clasifique como idiopático, la prescripción de terapias modificadoras de la enfermedad se recomienda como el estándar de tratamiento de la FPI.

La guía de práctica clínica de ATS/ ERS/JRS/ALAT recomienda el uso de pirfenidona o nintedanib para el tratamiento de la FPI en pacientes con pruebas de función pulmonar con deterioro leve a moderado, en una recomendación condicional en la que debe considerarse los costos del tratamiento y sus eventos adversos (1). Las guías del National Institute for Health and Care Excellence indican pirfenidona o nintedanib para pacientes con CVF entre 50 a $80 \%$ del predicho, recomendando suspender el tratamiento si hay evidencia de disminución en la CVF de 10\% o más, en cualquier momento dentro de un período de 12 meses $(41,42)$. La guía de la Sociedad Española de Neumología y Cirugía Torácica sugiere el tratamiento en pacientes con CVF igual o mayor de $50 \%$ y DLCO igual o mayor de $30 \%$ de sus predichos; si el paciente presenta deterioro de las pruebas de función pulmonar, debería evaluarse si se continua con el tratamiento, se cambia de medicamento o se combinan los medicamentos, mientras se es referido a un programa de trasplante pulmonar (43). La guía de la Asociación Latino Americana de Tórax (ALAT) sugiere el inicio del tratamiento en pacientes con diagnóstico reciente de la FPI, GVF mayor de $50 \%$ y DLCO mayor de $35 \%$ de sus predichos (44). Todas las guías recomiendan no usar inmunosupresores para el tratamiento de la FPI, sin embargo, los pacientes que los han estado recibiendo por largo plazo con tolerancia y estabilización de la enfermedad, podrían continuar esos tratamientos, aunque sería pertinente considerar un diagnóstico alternativo al de FPI $(1,44)$.

A la fecha, seis revisiones sistemáticas han realizado comparaciones indirectas entre pirfenidona y nintedanib, con distintos

Vol. 59(4): 369 - 385, 2018 
criterios de búsqueda y métodos estadísticos (45-50). En estas revisiones, ambos agentes han demostrado disminuir la tasa de declinamiento en la CVF; la proporción de pacientes que tienen una disminución igual o mayor de $10 \%$ en la CVF es similar para ambos medicamentos, pero, al analizar la CVF como una variable continua, el nintedanib obtiene una menor disminución (OR 0,67; IC 95\% 0,51-0,88) (46). El nintedanib también reduce la frecuencia de las exacerbaciones agudas $(46,47)$. Respecto a la mortalidad, solo la pirfenidona tiene un menor riesgo de mortalidad por todas las causas comparada a placebo, aunque sin diferencia en la comparación indirecta con el nintedanib $(46,49,50)$. La ausencia de un comparador común limita la validación de los resultados de las comparaciones indirectas; por otro lado, los estudios primarios no fueron diseñados para identificar la efectividad de los tratamientos en la mortalidad. A la fecha, ni la pirfenidona ni el nintedanib han demostrado una clara ventaja entre sí.

El deterioro en la CVF se ha establecido como el desenlace primario en los ensayos clínicos, debido a que es fácil de medir, es reproducible y se asocia a la mortalidad. Debido a que los criterios de selección para los ensayos clínicos fueron tan estrictos, las muertes registradas fueron pocas, por lo que no fue posible considerar la mortalidad como el desenlace primario. Desenlaces como las admisiones hospitalarias, no han sido utilizados como desenlace primario a pesar de estar relacionados con un mayor riesgo de muerte (51).

La decisión respecto a cuál tratamiento iniciar debe basarse en los potenciales eventos adversos, las comorbilidades y las preferencias del paciente. Los gastos generados por estos medicamentos en cada uno de los sistemas de salud deberían tomarse en cuenta, por lo que sería ideal tener información local de costo-efectividad de los tratamientos para la FPI (52). Por ejemplo, un análisis de costo-efectividad realizado en México en 2015, demostró costo-beneficio a favor de la pirfenidona sobre la triple terapia, mientras que un análisis comparativo en el sistema privado de salud brasileño en 2017 mostró una mejor relación costo-beneficio de la pirfenidona comparada con el nintedanib $(53,54)$.

La eficacia de un régimen de pirfenidona/nintedanib es desconocida. Un ensayo clínico reciente, evaluó la seguridad y la tolerabilidad de esta combinación y halló que los eventos adversos aumentaron y la concentración plasmática máxima de nintedanib tendió a ser menor cuando se añadió a pirfenidona (55). El estudio INJOURNEY evaluó la adición de pirfenidona a nintedanib durante 12 semanas, los casos de náuseas y vómitos aumentaron pero no hubo cambios en las concentraciones de nintedanib (56). En el análisis exploratorio, la disminución en la CVF fue menor en quienes usaron ambos medicamentos $(-13,3 \pm 17,4$ vs. $-40,9 \pm 31,4 \mathrm{~mL})$.

No existen suficientes datos sobre el uso de estos tratamientos en pacientes con comorbilidades significativas o con pruebas de función pulmonar leve o gravemente comprometidas. Considerando los criterios de inclusión de los estudios pivótales, solo un 27\% de los pacientes con FPI sería escogido para recibir tratamiento (57). Es necesario ponderar los beneficios y riesgos cuando se considere iniciar el tratamiento en individuos que fueron excluidos de los estudios (58). Dado el pronóstico ominoso de la enfermedad, su curso impredecible, la falta de terapias alternativas y la ausencia de una razón plausible para sospechar que el tratamiento no es útil en pacientes no incluidos en los estudios, es justificable la opinión de que el tratamiento debería extenderse a todos los pacientes para evitar una mayor progresión $(19,52)$.

El futuro de la FPI involucrará el desarrollo de terapias multinivel y terapias personalizadas, acorde a los mecanismos fisiopatológicos desarrollados por cada individuo (59). No obstante, debe señalarse que el manejo de la FPI va más allá del uso de terapias farmacológicas específicas, es necesario garantizar un enfoque de atención integrado y multidisciplinario para los pacientes, por 
medio de la evaluación en clínicas especializadas, el manejo de las comorbilidades y la rehabilitación pulmonar, intervenciones que pueden mejorar la calidad de vida y el cuidado paliativo de los síntomas $(60,61)$.

\section{CONCLUSIONES}

Ninguno de los fármacos ha demostrado un beneficio definitivo sobre la mortalidad en los estudios originales, aunque el análisis conjunto sugiere que pueden mejorar la supervivencia de los pacientes con FPI. Sus perfiles de seguridad son aceptables, pero deben tenerse en cuenta los potenciales eventos adversos al sopesar la decisión de iniciar alguna de las terapias.

El uso de estas terapias en poblaciones no incluidas en los estudios, siempre debe ser individualizado y ajustado a la realidad de cada sistema de salud.

Las terapias modificadoras de la enfermedad han renovado el panorama de la FPI, sin embargo, son solo una parte de todo el manejo integral que deben recibir estos pacientes.

\section{REFERENCIAS}

1. Raghu G, Rochwerg B, Zhang Y, Garcia CAC, Azuma A, Behr J, Brozek JL, Collard HR, Cunningham W, Homma S, Johkoh T, Martinez FJ, Myers J, Protzko SL, Richeldi L, Rind D, Selman M, Theodore A, Wells AU, Hoogsteden H, Schünemann HJ; American Thoracic Society; European Respiratory society; Japanese Respiratory Society; Latin American Thoracic Association. An official ATS/ERS/JRS/ALAT clinical practice guideline: Treatment of idiopathic pulmonary fibrosis. An update of the 2011 clinical practice guideline. Am J Respir Crit Care Med 2015; 192: e3-e19.

2. Richeldi L, Collard HR, Jones MG. Idiopathic pulmonary fibrosis. Lancet 2017; 389: 1941-1952.

3. Selman M, Pardo A. Revealing the pathogenic and aging-related mechanisms of the enigmatic idiopathic pulmonary fibrosis. an integral model. Am J Respir Crit Care Med 2014; 189: 1161-1172.

4. Wollin L, Wex E, Pautsch A, Schnapp G, Hostettler KE, Stowasser S, Kolb M. Mode of action of nintedanib in the treatment of idiopathic pulmonary fibrosis. Eur Respir J 2015; 45: 1434-1445.

5. Idiopathic Pulmonary Fibrosis Clinical Research Network, Raghu G, Anstrom KJ, King TE Jr, Lasky JA, Martinez FJ. Prednisone, azathioprine, and $\mathrm{N}$-acetyleysteine for pulmonary fibrosis. N Eng1 J Med 2012; 366: 1968-1977.

6. Xaubet A, Serrano-Mollar A, Ancochea J. Pirfenidone for the treatment of idiopathic pulmonary fibrosis. Expert Opin Pharmacother 2014; 15: 275-281.

7. Antoniou KM, Wuyts W, Wijsenbeek M, Wells AU. Medical therapy in idiopathic pulmonary fibrosis. Semin Respir Crit Care Med 2016; 37: 368-377.

8. Azuma A, Nukiwa T, Tsuboi E, Suga M, Abe S, Nakata K, Taguchi Y, Nagai S, Itoh H, Ohi M, Sato A, Kudoh S. Double-blind, placebo-controlled trial of pirfenidone in patients with idiopathic pulmonary fibrosis. Am J Respir Crit Care Med 2005; 171: 1040-1047.

9. Taniguchi H, Ebina M, Kondoh Y, Ogura T, Azuma A, Suga M, Taguchi Y, Takahashi H, Nakata K, Sato A, Takeuchi M, Raghu G, Kudoh S, Nukiwa T; Pirfenidone Clinical Study Group in Japan. Pirfenidone in idiopathic pulmonary fibrosis. Eur Respir J 2010; 35: 821-829.

10. Noble PW, Albera C, Bradford WZ, Costabel U, Glassberg MK, Kardatzke D, King TE Jr, Lancaster L, Sahn SA, Szwarcberg J, Valeyre D, du Bois RM; CAPACITY Study Group. Pirfenidone in patients with idiopathic pulmonary fibrosis (CAPACITY): two randomised trials. Lancet 2011; 377: 1760-1769.

11. King TE Jr, Bradford WZ, Castro-Bernardini S, Fagan EA, Glaspole I, Glassberǵ MK, Gorina E, Hopkins PM, Kardatzke D, Lancaster L, Lederer DJ, Nathan SD, Pereira CA, Sahn SA, Sussman R, Swigris JJ, Noble PW; ASCEND Study Group. A phase 3 trial of pirfenidone in patients with idiopathic pulmonary fibrosis. N Engl J Med 2014; 370: 2083-2092. 
12. Noble PW, Albera C, Bradford WZ, Costabel U, du Bois RM, Fagan EA, Fishman RS, Glaspole I, Glassberg MK, Lancaster L, Lederer DJ, Leff JA, Nathan SD, Pereira CA, Swigris JJ, Valeyre D, King TE Jr. Pirfenidone for idiopathic pulmonary fibrosis: analysis of pooled data from three multinational phase 3 trials. Eur Respir J 2016; 47: 243-253.

13. Nathan SD, Albera C, Bradford WZ, Costabel U, Glaspole I, Glassberg MK, Kardatzke DR, Daigl M, Kirchgaessler KU, Lancaster LH, Lederer DJ, Pereira CA, Swigris JJ, Valeyre D, Noble PW. Effect of pirfenidone on mortality: pooled analyses and meta-analyses of clinical trials in idiopathic pulmonary fibrosis. Lancet Respir Med 2017; 5: 33-41.

14. Ley B, Swignis J, Day B-M, Stauffer JL, Raimundo K, Chou W, Collard HR. Pirfenidone reduces respiratory-related hospitalizations in idiopathic pulmonary fibrosis. Am J Respir Crit Care Med 2017; 196: 756-761.

15. Aravena C, Labarea G, Venegas G, Arenas A, Rada G. Pirfenidone for idiopathic pulmonary fibrosis: A systematic review and meta-analysis. PloS One 2015; 10: e0136160.

16. Fisher M, Nathan SD, Hill C, Marshall J, Dejonckheere F, Thuresson PO, Maher TM. Predicting life expectancy for pirfenidone in idiopathic pulmonary fibrosis. J Manag Care Spec Pharm 2017; 23: S17-S24.

17. Albera C, Costabel U, Fagan EA, Glassberg MK, Gorina E, Lancaster L, Lederer DJ, Nathan SD, Spirig D, Swigris JJ. Efficacy of pirfenidone in patients with idiopathic pulmonary fibrosis with more preserved lung function. Eur Respir J 2016; 48: 843851.

18. Nathan SD, Albera C, Bradford WZ, Costabel U, du Bois RM, Fagan EA, Fishman RS, Glaspole I, Glassberg MK, Glasscock KF, King TE Jr, Lancaster L, Lederer DJ, Lin Z, Pereira CA, Swigris JJ, Valeyre D, Noble PW, Wells AU. Effect of continued treatment with pirfenidone following clinically meaningful declines in forced vital capacity: analysis of data from three phase 3 trials in patients with idiopathic pulmonary fibrosis. Thorax 2016; 71: 429-435.
19. Torrisi SE, Pavone M, Vancheri A, Vancheri C. When to start and when to stop antifibrotic therapies. Eur Respir Rev 2017; 26: pii: 170053.

20. Costabel U, Bendstrup E, Cottin V, Dewint P, Egan JJ, Ferguson J, Groves R, Hellström PM, Kreuter M, Maher TM, Molina-Molina M, Nordlind K, Sarafidis A, Vancheri C. Pirfenidone in idiopathic pulmonary fibrosis: expert panel discussion on the management of drug-related adverse events. Adv Ther 2014; 31: 375-391.

21. Lancaster L, Albera C, Bradford WZ, Costabel U, du Bois RM, Fagan EA, Fishman RS, Glaspole I, Glassberg MK, King TE Jr, Lederer DJ, Lin Z, Nathan SD, Pereira CA, Swigris JJ, Valeyre D, Noble PW. Safety of pirfenidone in patients with idiopathic pulmonary fibrosis: integrated analysis of cumulative data from 5 clinical trials. BMJ Open Respir Res 2016; 3: e000105.

22. Arai T, Inoue $Y$, Sasaki $Y$, Tachibana $K$, Nakao K, Sugimoto C, Okuma T, Akira M, Kitaichi M, Hayashi S. Predictors of the clinical effects of pirfenidone on idiopathic pulmonary fibrosis. Respir Investig 2014; 52: 136-143.

23. Behr J, Bendstrup E, Crestani B, Günther A, Olschewski H, Sköld CM, Wells A, Wuyts W, Koschel D, Kreuter M, Wallaert B, Lin CY, Beck J, Albera C. Safety and tolerability of acetyleysteine and pirfenidone combination therapy in idiopathic pulmonary fibrosis: a randomised, double-blind, placebo-controlled, phase 2 trial. Lancet Respir Med 2016; 4: 445-453.

24. Keating GM. Nintedanib: A review of its use in patients with idiopathic pulmonary fibrosis. Drugs 2015; 75: 1131-1140.

25. Richeldi L, Costabel U, Selman M, Kim DS, Hansell DM, Nicholson AG, Brown KK, Flaherty KR, Noble PW, Raghu G, Brun M, Gupta A, Juhel N, Klüglich M, du Bois RM. Efficacy of a tyrosine kinase inhibitor in idiopathic pulmonary fibrosis. $\mathrm{N}$ Engl J Med 2011; 365: 1079-1087.

26. Richeldi L, du Bois RM, Raghu G, Azuma A, Brown KK, Costabel U, Cottin V, Flaherty KR, Hansell DM, Inoue Y, Kim DS, Kolb M, Nicholson AG, Noble PW, Selman M, Taniguchi H, Brun M, Le Maulf F, Girard M, Stowasser S, Schlenker-Herceg $\mathrm{R}$, 
Disse B, Collard HR; INPULSIS Trial Investigators. Efficacy and safety of nintedanib in idiopathic pulmonary fibrosis. N Engl J Med 2014; 370: 2071-2082.

27. Costabel U, Inoue Y, Richeldi L, Collard HR, Tschoepe I, Stowasser S, Azuma A. Efficacy of nintedanib in idiopathic pulmonary fibrosis across prespecified subgroups in INPULSIS. Am J Respir Crit Care Med 2016; 193: 178-185.

28. Raghu G, Wells AU, Nicholson AG, Richeldi L, Flaherty KR, Le Maulf F, Stowasser S, Schlenker-Herceg R, Hansell DM. Effect of nintedanib in subgroups of idiopathic pulmonary fibrosis by diagnostic criteria. Am J Respir Crit Care Med 2017; 195: 78-85.

29. Kolb M, Richeldi L, Behr J, Maher TM, Tang W, Stowasser S, Hallmann C, du Bois RM. Nintedanib in patients with idiopathic pulmonary fibrosis and preserved lung volume. Thorax 2017; 72: 340-346.

30. Richeldi L, Cottin V, du Bois RM, Selman M, Kimura T, Bailes Z, Schlenker-Herceg R, Stowasser S, Brown KK. Nintedanib in patients with idiopathic pulmonary fibrosis: Combined evidence from the TOMORROW and INPULSIS(®) trials. Respir Med 2016; 113: 74-79.

31. Richeldi L, Kreuter M, Selman M, Crestani B, Kirsten AM, Wuyts WA, Xu Z, Bernois K, Stowasser S, Quaresma M, Costabel U. Long-term treatment of patients with idiopathic pulmonary fibrosis with nintedanib: results from the TOMORROW trial and its open-label extension. Thorax 2018; 73: 581-583.

32. Wuyts WA, Kolb M, Stowasser S, Stansen W, Hugǵins JT, Raghu G. First data on efficacy and safety of nintedanib in patients with idiopathic pulmonary fibrosis and forced vital capacity of $\leq 50 \%$ of predicted value. Lung 2016; 194: 739-743.

33. Corte T, Bonella F, Crestani B, Demedts MG, Richeldi L, Coeck C, Pelling K, Quaresma M, Lasky JA. Safety, tolerability and appropriate use of nintedanib in idiopathic pulmonary fibrosis. Respir Res 2015; 16 : 116.

34. Harari S, Caminati A. Idiopathic pulmonary fibrosis: from clinical trials to reallife experiences. Eur Respir Rev 2015; 24: 420-427.
35. Ogura T, Azuma A, Inoue $\mathrm{Y}$, Taniguchi H, Chida K, Bando M, Niimi Y, Kakutani S, Suga M, Sugiyama Y, Kudoh S, Nukiwa T. All-case post-marketing surveillance of 1371 patients treated with pirfenidone for idiopathic pulmonary fibrosis. Respir Investig 2015; 53: 232-241.

36. Hughes G, Toellner H, Morris H, Leonard C, Chaudhuri N. Real world experiences: Pirfenidone and nintedanib are effective and well tolerated treatments for idiopathic pulmonary fibrosis. J Clin Med 2016; 5: pii: E78.

37. Oltmanns U, Kahn N, Palmowski K, Träger A, Wenz H, Heussel CP, Schnabel PA, Puderbach M, Wiebel M, Ehlers-Tenenbaum S, Warth A, Herth FJ, Kreuter M. Pirfenidone in idiopathic pulmonary fibrosis: reallife experience from a German tertiary referral center for interstitial lung diseases. Respiration 2014; 88: 199-207.

38. Bonella F, Kreuter M, Hagmeyer L, Neurohr C, Keller C, Kohlhaeufl MJ, MüllerQuernheim J, Milger K, Prasse A; German Nintedanib Compassionate Use Consortium. Insights from the German compassionate use program of nintedanib for the treatment of idiopathic pulmonary fibrosis. Respiration 2016; 92: 98-106.

39. Galli JA, Pandya A, Vega-Olivo M, Dass C, Zhao H, Criner GJ. Pirfenidone and nintedanib for pulmonary fibrosis in clinical practice: Tolerability and adverse drug reactions. Respirology 2017; 22: 1171-1178.

40. Tabaj GC, Sivori M, Cornejo L, Plotquin M. Experiencia en la Argentina del Programa de uso compasivo con nintedanib en el tratamiento de la Fibrosis Pulmonar Idiopática. Rev Am Med Respir 2017; 17: 126-130.

41. National Institute for Health and Care Excellence. Nintedanib for treating idiopathic pulmonary fibrosis. NICE Guidance and guidelines [Internet] 2016 [cited $2018 \mathrm{Feb}$ 11]. Available from: URL:https://www.nice. org.uk/guidance/ta379

42. National Institute for Health and Care Excellence. Pirfenidone for treating idiopathic pulmonary fibrosis. NICE Guidance and guidelines [Internet] 2018 [cited 2018 Feb 11]. Available from: URL:https://www. nice.org.uk/guidance/ta504 
43. Xaubet A, Molina-Molina M, Acosta O, Bollo E, Castillo D, Fernández-Fabrellas E, Rodríguez-Portal JA, Valenzuela C, Ancochea J. Guidelines for the medical treatment of idiopathic pulmonary fibrosis. Arch Bronconeumol 2017; 53: 263-269.

44. Asociación Latino Americana de Tórax. Recomendaciones para el diagnóstico y tratamiento de la Fibrosis Pulmonar Idiopática [Internet] 2015 [cited 2018 Jan 9]. Available from: URL:https://alatorax.org/ es/recomendaciones/recomendaciones-para-el-diagnostico-y-tratamiento-de-la-fibrosis-pulmonar-idiopatica-2015

45. Loveman E, Copley VR, Colquitt JL, Scott DA, Cleǵg AJ, Jones J, O'Reilly KM, Singh S, Bausewein C, Wells A. The effectiveness and cost-effectiveness of treatments for idiopathic pulmonary fibrosis: systematic review, network meta-analysis and health economic evaluation. BMC Pharmacol Toxicol 2014; 15: 63.

46. Loveman E, Copley VR, Scott DA, Colquitt JL, Clegg AJ, O'Reilly KM. Comparing new treatments for idiopathic pulmonary fibrosis--a network meta-analysis. BMC Pulm Med 2015; 15: 37.

47. Rogliani P, Calzetta L, Cavalli F, Matera MG, Cazzola M. Pirfenidone, nintedanib and $\mathrm{N}$-acetylcysteine for the treatment of idiopathic pulmonary fibrosis: A systematic review and meta-analysis. Pulm Pharmacol Ther 2016; 40: 95-103.

48. Canestaro WJ, Forrester SH, Raghu G, Ho L, Devine BE. Drug treatment of idiopathic pulmonary fibrosis: Systematic review and network meta-analysis. Chest 2016; 149: 756-766.

49. Rochwerg B, Neupane B, Zhang Y, Garcia CC, Raghu G, Richeldi L, Brozek J, Beyene $J$, Schünemann $H$. Treatment of idiopathic pulmonary fibrosis: a network metaanalysis. BMC Med 2016; 14: 18.

50. Fleetwood K, McCool R, Glanville J, Edwards SC, Gsteiger S, Daigl M, Fisher M. Systematic review and network metaanalysis of idiopathic pulmonary fibrosis treatments. J Manag Care Spec Pharm 2017; 23: S5-S16.

51. Durheim MT, Collard HR, Roberts RS, Brown KK, Flaherty KR, King TE Jr, Palmer SM, Raghu G, Snyder LD, Anstrom
KJ, Martinez FJ; IPFnet investigators. Association of hospital admission and forced vital capacity endpoints with survival in patients with idiopathic pulmonary fibrosis: analysis of a pooled cohort from three clinical trials. Lancet Respir Med 2015; 3 : 388-396.

52. King CS, Nathan SD. POINT: Should all patients with idiopathic pulmonary fibrosis, even those with more than moderate impairment, be treated with nintedanib or pirfenidone? Yes. Chest 2016; 150: 273-275.

53. Pozo L, Paladio-Hernández J. Cost-effective evaluation of pirfenidone for treating idiopathic pulmonary fibrosis in Mexico. Value Health 2015; 18: A840-A841.

54. Ho R, Rufino C, Lisondo C, Alves M. Costeffectiveness analysis of pirfenidone versus nintedanib on the treatment of idiopathic pulmonary fibrosis. Value Health $2017 ; 20$ : A893-A894.

55. Ogura T, Taniguchi $H$, Azuma $A$, Inoue $Y$, Kondoh Y, Hasegawa Y, Bando M, Abe S, Mochizuki Y, Chida K, Klüglich M, Fujimoto T, Okazaki K, Tadayasu Y, Sakamoto W, Sugiyama Y. Safety and pharmacokinetics of nintedanib and pirfenidone in idiopathic pulmonary fibrosis. Eur Respir J 2015; 45: 1382-1392.

56. Vancheri C, Kreuter M, Richeldi L, Ryerson CJ, Valeyre D, Grutters JC, Wiebe S, Stansen W, Quaresma M, Stowasser S, Wuyts WA; INJOURNEY Trial Investigators. Nintedanib with add-on pirfenidone in idiopathic pulmonary fibrosis. Results of the INJOURNEY trial. Am J Respir Crit Care Med 2018; 197: 356-363.

57. Nathan SD, Shlobin OA, Weir N, Ahmad S, Kaldjob JM, Battle E, Sheridan MJ, du Bois RM. Long-term course and prognosis of idiopathic pulmonary fibrosis in the new millennium. Chest 2011; 140: 221-229.

58. Brown KK. COUNTERPOINT: Should all patients with idiopathic pulmonary fibrosis, even those with more than moderate impairment, be treated with nintedanib or pirfenidone? No. Chest 2016; 150: 276-278.

59. Ferrara G, Luppi F, Birring SS, Cerri S, Caminati A, Sköld M, Kreuter M. Best supportive care for idiopathic pulmonary fibrosis: current gaps and future directions. Eur Respir Rev 2018; 27: pii: 170076. 
60. Kulkarni T, Willoughby J, Acosta Lara MDP, Kim Y-I, Ramachandran R, Alexander CB, Luckhardt T, Thannickal VJ, de Andrade JA. A bundled eare approach to patients with idiopathic pulmonary fibrosis improves transplant-free survival. Respir Med 2016; 115: 33-38.
61. Buendía-Roldán I, Mejía M, Navarro G, Selman M. Idiopathic pulmonary fibrosis: Clinical behavior and aǵing associated comorbidities. Respir Med 2017; 129: 46-52. 